\title{
Spin Pair Correlation of the ANNNI Chain in a Field
}

\author{
Nelson Alves Jr. and Carlos S. O. Yokoi \\ Instituto de Física, Universidade de São Paulo, \\ Caixa Postal 66318, 05315-970 São Paulo, SP, Brazil
}

Received on 10 October, 2000

\begin{abstract}
We study the spin pair correlation function of the one-dimensional Ising model with competing nearest and next-nearest neighbor interactions, or ANNNI chain, in the presence of an external field. Of particular interest are the disorder lines where exponential decay of the spin pair correlation changes from monotonic to oscillatory. We extend previous studies for higher field values and obtain asymptotic expressions for disorder lines at low temperatures. We also observe reentrant disorder lines.
\end{abstract}

\section{Introduction}

The axial next-nearest neighbor Ising (ANNNI) model is an example of a frustrated system [1] displaying modulated order at low temperatures in two and three dimensions $[2,3]$. In one dimension the model is exactly solvable and has been investigated in zero field [4-10], in the presence of a field $[11,12]$ and at zero temperature $[13,14]$. Of particular interest is the exponential decay of the spin pair correlation function which may be modulated by an oscillatory factor with a temperature- and field-dependent wave number. The line in the phase diagram that separates the region with zero wave number from non-zero wave number has been called the disorder line $[7,8]$. The main purpose of this paper is to further investigate the ANNNI chain in a field and examine some aspects of the spin pair correlation not considered previously.

The energy of the model can be written

$$
E=-\sum_{i=1}^{N}\left(J_{1} \sigma_{i} \sigma_{i+1}+J_{2} \sigma_{i} \sigma_{i+2}+H \sigma_{i}\right)+E_{0},
$$

where $\sigma_{i}= \pm 1$ and the periodic boundary condition $\sigma_{i+N}=\sigma_{i}$ is assumed. $J_{1}$ and $J_{2}$ are the nearest and the next-nearest neighbor interactions, respectively, and $H$ is the magnetic field. For low temperature numerical calculations it is convenient to take $E_{0}$ to be the ground state energy, since this choice avoids the divergence of the eigenvalues of the transfer matrix. We will restrict our discussion to ferromagnetic nearest neighbor interaction $\left(J_{1}>0\right)$, antiferromagnetic next-nearest neighbor interaction $\left(J_{2}<0\right)$ and positive field $(H>0)$. The ratio $-J_{2} / J_{1}$ measures the competition between nearest and next-nearest neighbor interactions. The zero temperature phase diagram in the plane $H$ versus $-J_{2} / J_{1}$ is shown in Fig. 1 . There are only two phases: In the ferromagnetic phase all the spins point up, whereas in the antiphase state two up spins alternate with two down spins $[13,14]$. We shall principally consider parameter values yielding a ferromagnetic ground state with

$$
E_{0}=-\sum_{i=1}^{N}\left(J_{1}+J_{2}+H\right)
$$

The solution of this problem can be formulated in terms of the transfer matrix $\mathbf{V}$ with elements $[9,12,15]$

$$
\left\langle\sigma_{1} \sigma_{2}|\mathbf{V}| \sigma_{3} \sigma_{4}\right\rangle=\delta_{\sigma_{2}, \sigma_{3}} x^{1-\sigma_{1} \sigma_{2}} y^{1-\sigma_{1} \sigma_{4}} z^{1-\sigma_{1}},
$$

where

$$
x=e^{-\beta J_{1}}, \quad y=e^{-\beta J_{2}}, \quad z=e^{-\beta H},
$$

with $\beta=1 / k_{\mathrm{B}} T, k_{\mathrm{B}}$ being Boltzmann's constant and $T$ the absolute temperature. The eigenvalues are given by the secular equation

$$
f(\lambda)=a_{0} \lambda^{4}+4 a_{1} \lambda^{3}+6 a_{2} \lambda^{2}+4 a_{3} \lambda+a_{4}=0,
$$

where

$$
\begin{gathered}
a_{0}=1, \quad a_{1}=-\frac{1}{4}\left(1+z^{2}\right), \quad a_{2}=\frac{1}{6}\left(1-x^{4}\right) z^{2}, \\
a_{3}=-\frac{1}{4} x^{4} z^{2}\left(1+z^{2}\right) w, \quad a_{4}=-x^{4} z^{4} w^{2}
\end{gathered}
$$

with

$$
w=y^{4}-1
$$

The nature of these eigenvalues is determined by the discriminant [16] of the quartic equation (5),

$$
\Delta=\left(a_{0} a_{4}-4 a_{1} a_{3}+3 a_{2}^{2}\right)^{3}
$$




$$
-27\left(a_{0} a_{2} a_{4}-a_{0} a_{3}^{2}-a_{1}^{2} a_{4}+2 a_{1} a_{2} a_{3}-a_{2}^{3}\right)^{2} .
$$

The condition for two real and two complex roots is $\Delta<0$, and that for four real or four complex roots is $\Delta>0$ [16]. Since $f( \pm \infty)=\infty$ and $f(0)<0$, there will always be two real roots with the largest root being real and positive. Moreover, since $f^{\prime}(\lambda)<0$ for $\lambda<0$, if the second largest root in magnitude is real then it will be positive. Let $\lambda_{1}$ denote the largest eigenvalue and $\lambda_{2}$ the second largest eigenvalue in magnitude. In the thermodynamic limit $N \rightarrow \infty$ the spin pair correlation at large distances $r \gg 1$ has the asymptotic form [12]

$$
\left\langle\sigma_{i} \sigma_{i+r}\right\rangle \sim m^{2}+A e^{-r / \xi} \cos q r,
$$

where $m=\left\langle\sigma_{i}\right\rangle$ is the magnetization, $A$ is the amplitude, $\xi$ is the correlation length and $q$ is the wave number, where

$$
\xi=\ln \frac{\lambda_{1}}{\left|\lambda_{2}\right|}, \quad q=\arg \lambda_{2} .
$$

If $\lambda_{2}$ is real then $q=0$ and the correlation will have a monotonic exponential decay, whereas if $\lambda_{2}$ is complex there will be an oscillatory decay with wave number $q$. The changeover from monotonic to oscillatory decay defines the disorder line $[7,8]$ or Lifshitz condition [10]. Therefore the condition for the disorder line is the vanishing of the discriminant $\Delta$ given by (8).

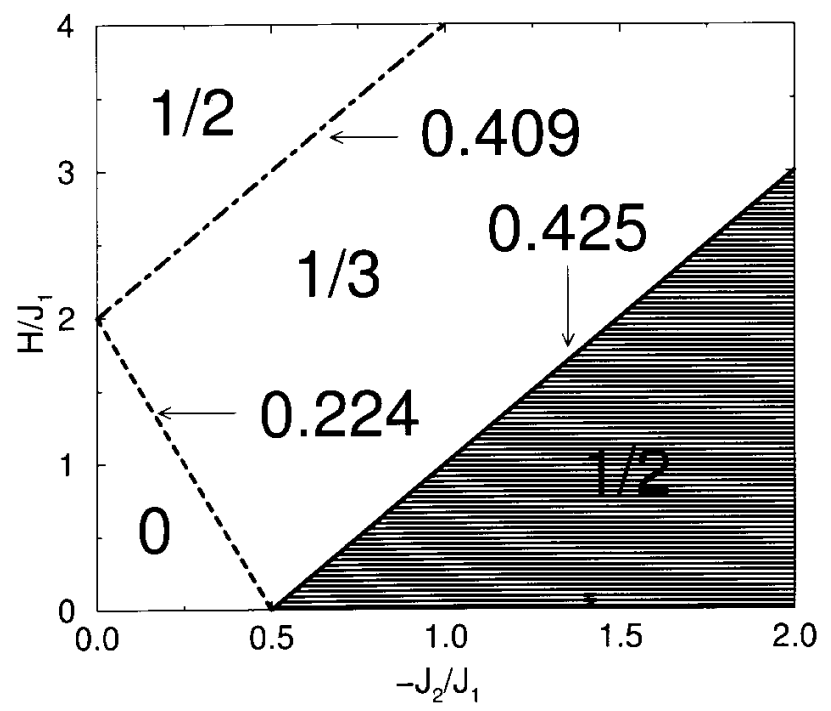

Figure 1. Zero temperature phase diagram of the ANNNI chain in a magnetic field. The solid line $-J_{2} / J_{1}=1 / 2+$ $H / 2 J_{1}$ separates the ferromagnetic phase from the antiphase (shaded region). The numbers indicate the limiting values taken by the wave number $q / \pi$ as $T \rightarrow 0$ inside the regions and over the lines separating them. The disorder lines intersect the $T=0$ axis for $H / J_{1}<2$ on the dashed line $-J_{2} / J_{1}=1 / 2-H / 4 J_{1}$. The dot-dashed line $-J_{2} / J_{1}=H / 2 J_{1}-1$ separates regions with different limiting wave number values for $H / J_{1}>2$.

\section{Numerical Results}

Let us first consider field values $H / J_{1}<2$. Fig. 2 shows disorder lines in the plane of temperature $T$ versus $-J_{2} / J_{1}$ for various fixed values of the magnetic field $H$. The wave number of the spin pair correlation (9) is zero to the left of the disorder line and non-zero to the right. The behavior of the wave number $q$ as a function of temperature $T$ is illustrated in Fig. 3 for the field $H / J_{1}=1.5$ and various fixed values of $-J_{2} / J_{1}$. An interesting aspect of these graphs [12] is that for $0.125 \leq-J_{2} / J_{1} \leq 1.25$ the limiting value of wave number $q$ as $T \rightarrow 0$ is non-zero despite the ground state being ferromagnetic. There is no contradiction, however, since in the ferromagnetic phase $m \rightarrow 1$ and $\xi \rightarrow 0$ as $T \rightarrow 0$. Thus the second term in the right hand side of Eq. (9) vanishes and the wave number is not relevant as far as the ground state ordering is concerned. On the other hand, in the antiphase $m \rightarrow 0$ and $\xi \rightarrow \infty$ as $T \rightarrow 0$. Therefore it is the first term in the right hand side of Eq. (9) that vanishes and the wave number $q=\pi / 2$ correctly describes the antiphase state [12]. In the zero temperature phase diagram of Fig. 1 we have indicated the limiting values taken by the wave number $q$ as $T \rightarrow 0$. Another interesting feature is the reentrant behavior exhibited by the disorder lines for large enough fields: As the temperature is lowered the wave number first vanishes but at still lower temperatures it becomes non-zero again, as shown in Fig. 3 for the case $-J_{2} / J_{1}=0.08$. We found numerically that the reentrant behavior of disorder lines is present for all fields $H / J_{1}>1.333$.

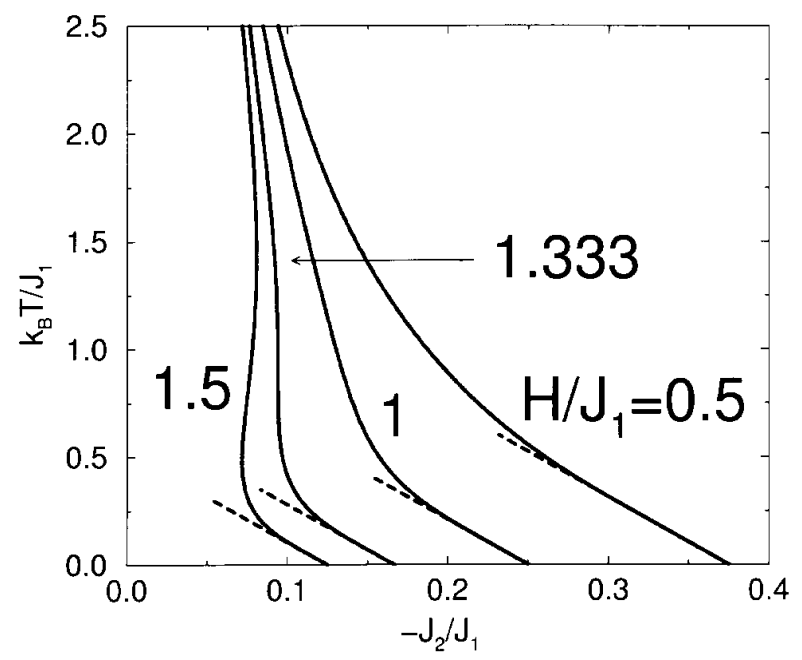

Figure 2. Disorder lines for various fixed values of the magnetic field $H / J_{1}<2$. The wave number is zero to the left and non-zero to the right of these lines. The straight dashed lines correspond to the asymptotic results for $T \rightarrow 0$. For $H / J_{1}>1.333$ the disorder lines display reentrant behavior. 


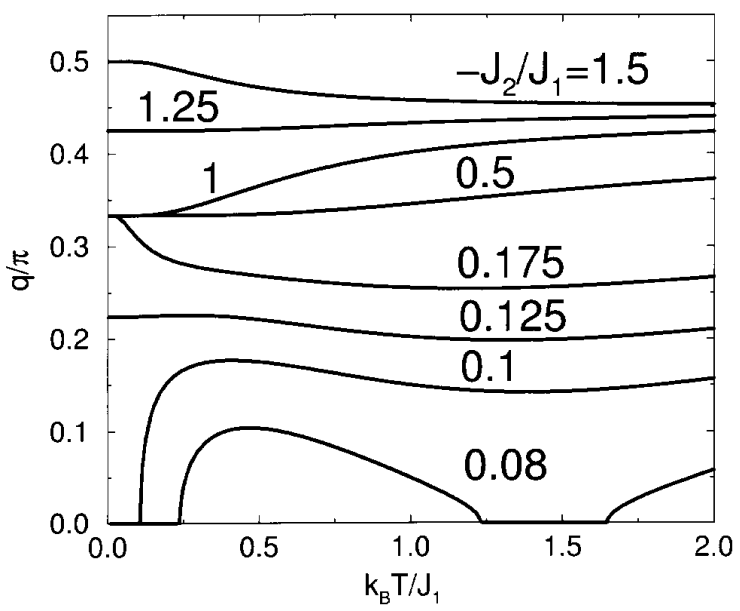

Figure 3. Wave number $q$ as a function of temperature $T$ for $H / J_{1}=1.5$ and various fixed values of $-J_{2} / J_{1}$. The graph for $-J_{2} / J_{1}=0.08$ shows the vanishing of the wave number at intermediate temperatures corresponding to the reentrant behavior of the disorder line.

We will now consider field values $H / J_{1} \geq 2$. Fig. 4 shows the disorder lines in the plane of temperature $T$ versus $-J_{2} / J_{1}$ for various fixed values of the field $H$. The wave number of the spin pair correlation (9) is zero to the left of the disorder line and non-zero to the right. We observe that in contrast to the case $H / J_{1}<2$, all disorder lines tend to $-J_{2} / J_{1}=0$ as $T \rightarrow 0$. In Fig. 5 the graphs of the wave number $q$ as a function of temperature $T$ are shown for the field $H / J_{1}=3$ and several values of $-J_{2} / J_{1}$. The limiting value of the wave number $q$ as $T \rightarrow 0$ is always non-zero. Also, although it is outside the range of temperatures shown, the wave number for $-J_{2} / J_{1}=0.01$ vanishes only in some temperature interval, reflecting the reentrant behavior of the disorder line. Finally we observe that, no matter the field strength $H$, all the disorder lines tend to the $T$ axis as $T \rightarrow \infty$.

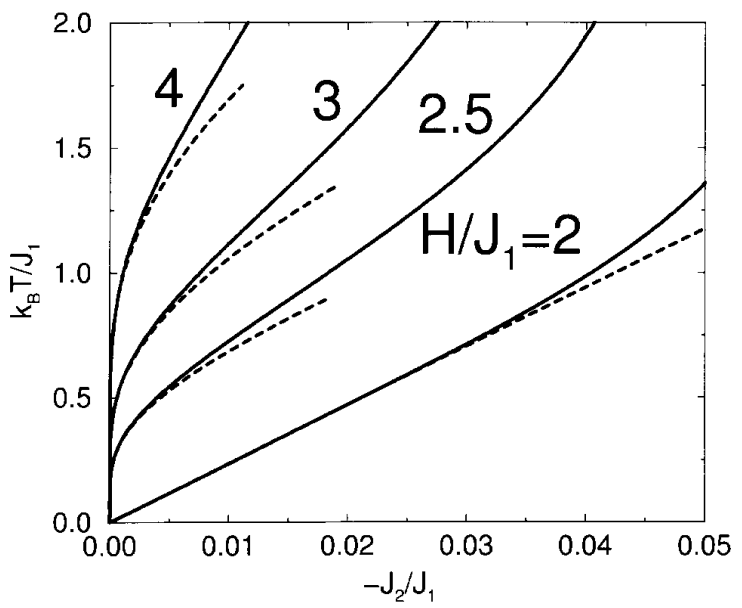

Figure 4. Disorder lines for various fixed values of the magnetic field $H / J_{1} \geq 2$. The wave numbers are zero to the left and non-zero to the right of these lines. The dashed lines correspond to the asymptotic results for $T \rightarrow 0$. All the disorder lines display reentrant behavior.

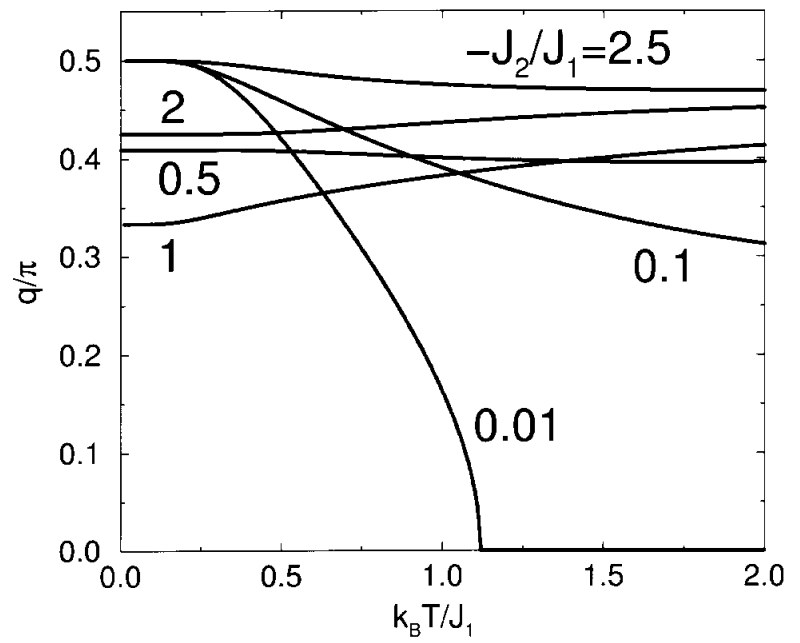

Figure 5. Wave number $q$ as a function of temperature $T$ for $H / J_{1}=3$ and various fixed values of $-J_{2} / J_{1}$. For $-J_{2} / J_{1}=0.01$ the wave number vanishes at intermediate temperatures corresponding to the reentrant behavior of the disorder line, but the temperature value at which the wave number assumes non-zero value again is off the scale and cannot be seen.

\section{Asymptotic Results}

In this section we obtain asymptotic expressions for disorder lines at low temperatures. Since $x \rightarrow 0$ and $z \rightarrow 0$ as $T \rightarrow 0$, the discriminant (8) is given to leading order by

$$
\begin{aligned}
\Delta \sim & \frac{z^{12}}{6912}\left[4\left(1-12 x^{4} w^{2}-3 \frac{x^{4} w}{z^{2}}\right)^{3}\right. \\
& \left.-\left(2-27 \frac{x^{4} w^{2}}{z^{2}}-9 \frac{x^{4} w}{z^{2}}\right)^{2}\right] .
\end{aligned}
$$

We first assume that $w \rightarrow \infty$ as $T \rightarrow 0$. Then the discriminant (11) can vanish only if $x^{4} w^{2}=O\left(z^{2}\right)$ and we have

$$
\Delta \sim \frac{z^{12}}{6912}\left[4-\left(2-27 \frac{x^{4} w^{2}}{z^{2}}\right)^{2}\right] .
$$

The disorder line condition $\Delta=0$ gives $x^{4} w^{2} / z^{2}=$ $4 / 27$ or

$$
-\frac{J_{2}}{J_{1}} \sim \frac{1}{2}-\frac{H}{4 J_{1}}-\frac{k_{\mathrm{B}} T}{8 J_{1}} \ln \left(\frac{27}{4}\right) .
$$

This result, valid only for $H / J_{1}<2$, implies that the disorder lines meet the $T=0$ axis with field independent slope $-\ln (27 / 4) / 8=-0.238 \ldots$ at

$$
-\frac{J_{2}}{J_{1}}=\frac{1}{2}-\frac{H}{4 J_{1}}
$$

in good agreement with the numerical results of Fig. 2. The above equation also corresponds to the straight line separating the region with $q / \pi=0$ from the region 
with $q / \pi=1 / 3$ in the zero temperature phase diagram of Fig. 1. Let us next assume that $w \rightarrow 0$ as $T \rightarrow 0$. We then have

$$
\Delta \sim \frac{z^{12}}{6912}\left[4\left(1-3 \frac{x^{4} w}{z^{2}}\right)^{3}-\left(2-9 \frac{x^{4} w}{z^{2}}\right)^{2}\right] .
$$

The disorder line condition $\Delta=0$ gives $x^{4} w / z^{2}=1 / 4$ or

$$
-\frac{J_{2}}{J_{1}} \sim \frac{k_{\mathrm{B}} T}{16 J_{1}} \exp \left(\frac{4 J_{1}-2 H}{k_{\mathrm{B}} T}\right) .
$$

This result, valid for $H / J_{1}>2$, implies exponential approach of the disorder lines to the temperature axis as $T \rightarrow 0$, in good agreement with the numerical results of Fig. 4. Finally, the case where $w$ remains finite as $T \rightarrow 0$ should correspond to $H / J_{1}=2$. Then $x^{4} / z^{2}=1$ and we have

$$
\Delta \sim \frac{z^{12}}{6912}\left[4(1-3 w)^{3}-\left(2-27 w^{2}-9 w\right)^{2}\right] .
$$

The disorder line condition $\Delta=0$ gives $w=5 / 27$. Therefore

$$
-\frac{J_{2}}{J_{1}} \sim \frac{1}{4} \ln \left(\frac{32}{27}\right) \frac{k_{\mathrm{B}} T}{J_{1}} .
$$

This result shows that the disorder line approaches the origin as $T \rightarrow 0$ with slope $\ln (32 / 27) / 4=0.04247 \ldots$, in good agreement with the numerical result of Fig. 4.

\section{Summary}

We have extended previous work on the ANNNI chain in a field [12] by considering the spin pair correlations for all field values. We have shown that the disorder lines have different low temperature behavior for $H / J_{1}<2, H / J_{1}=2$ and $H / J_{1}>2$. Numerical results were confirmed by asymptotic calculations for $T \rightarrow 0$. We have also shown that the disorder lines have reentrant behavior for $H / J_{1}>1.333$.

\section{Acknowledgments}

The authors acknowledge financial support from Brazilian agencies Fundação de Amparo à Pesquisa do Estado de São Paulo (FAPESP) and Conselho Nacional de Desenvolvimento Científico e Tecnológico $(\mathrm{CNPq})$.

\section{References}

[1] G. Toulouse, Commun. Phys. 2, 115 (1977).

[2] W. Selke, Phys. Rep. 170, 213 (1988).

[3] J. M. Yeomans, in Solid State Physics, edited by H. Ehrenreich and D. Turnbull (Academic Press, San Diego (CA), 1988), Vol. 41, pp. 151-200.

[4] E. W. Montroll, J. Chem. Phys. 10, 61 (1942).

[5] J. S. Marsh, Phys. Rev. 145, 251 (1966).

[6] J. F. Dobson, J. Math. Phys. 10, 40 (1969).

[7] J. Stephenson, Phys. Rev. B 1, 4405 (1970).

[8] J. Stephenson, Can. J. Phys. 48, 1724 (1970).

[9] S. Katsura and M. Ohminami, J. Phys. A 5, 95 (1972).

[10] R. M. Hornreich, R. Liebmann, H. G. Schuster, and W. Selke, Z. Phys. B 35, 91 (1979).

[11] P. Rujan, W. Selke, and G. V. Uimin, Z. Phys. B 53, 221 (1983).

[12] N. Bhattacharyya and S. Dasgupta, J. Phys. A 24, 3927 (1991).

[13] T. Morita and T. Horiguchi, Phys. Lett. 38A, 223 (1972).

[14] C. S. O. Yokoi, M. D. Coutinho-Filho, and S. R. Salinas, Phys. Rev. B 24, 4047 (1981).

[15] F. A. Kasssan-Ogly, E. V. Kormil'tsev, V. E. Naǐsh, and I. V. Sagaradze, Fiz. Tverd. Tela (Leningrad) 31, 43 (1989), [Sov. Phys. — Solid State 31, 939 (1989)].

[16] H. W. Turnbull, Theory of Equations (Oliver and Boyd, Edinburgh and London, 1963). 